\title{
THE SINGULAR IDEAL AND RADICALS
}

\section{MIGUEL FERRERO and EDMUND R. PUCZYLOWSKI}

(Received 9 November 1995; revised 2 December 1997)

Communicated by L. Kovács

\begin{abstract}
Some properties of the singular ideal are established. In particular its behaviour when passing to one-sided ideals is studied. Obtained results are applied to study some radicals related to the singular ideal. In particular a radical $S$ such that for every ring $R, S(R)$ and $R / S(R)$ are close to being a singular ring and a non-singular ring, respectively, is constructed.
\end{abstract}

1991 Mathematics subject classification (Amer. Math. Soc.): Primary 16D80, 16N60, 16N80. Keyw'ords and phrases: singular ideal, radical classes.

\section{Introduction}

Studying properties of rings one can usually say more assuming that the considered rings are either singular or non-singular. It is natural to ask whether one can reduce studies of arbitrary rings to these two particular cases. In this paper we examine a radical approach to the question namely, we study whether one can construct a radical $S$ such that for every ring $R, S(R)$ is not far from being a singular ring whereas $R / S(R)$ is close to being a non-singular ring.

In the first two sections of the paper we study properties of the singular ideal of a ring. In particular we study how it behaves when passing to ideals or one-sided ideals. Obtained properties are applied in the third section to find a radical of the above specified type. The radical seems to be quite satisfactory. It satisfies the required conditions, is quite regular (it is an $N$-radical in the sense of A.D.Sands [14]) and related to some other classical radicals (it contains the prime radical and is contained in the strongly prime radical). We study also some related radicals. In particular we

This research was partially supported by Conselho Nacional de Desenvolvimento Científico e Tecnológico (CNPq), Brazil

(C) 1998 Australian Mathematical Society $0263-6115 / 98 \$ A 2.00+0.00$ 
show that the class of rings (cf. [3] and the papers quoted therein) whose all prime homomorphic images are non-singular is an $N$-radical class.

In the last section we obtain some results on the singular ideal of rings satisfying the ascending chain condition on principal right ideals.

Some of the ideas of this paper are contained in the unpublished paper [4].

All rings considered in this paper are associative but, unless otherwise stated, are not assumed to have identity. The ring obtained from a ring $R$ by adjoining an identity will be denoted by $R^{*}$.

To denote that $I$ is an ideal (right ideal, left ideal) of a ring $R$ we write $I \triangleleft R$ $(I<r, R, I<, R)$.

Given a subset $S$ of a right (left) $R$-module $M$, the annihilator $\operatorname{ann}_{R}(S)$ of $S$ in $R$ is defined as $\{r \in R \mid S r=0\}(\{r \in R \mid r S=0\})$. If $m \in M$, then $\operatorname{ann}_{R}(\{m\})$ is denoted by $\operatorname{ann}_{R}(m)$. In case when $M=R_{R}$ (respectively $M={ }_{R} R$ ), $\operatorname{ann}_{R}(S)$ is denoted by $r_{R}(S)$ (respectively $l_{R}(S)$ ) (if the context is clear, just by $r(S)$ or $l(S)$ ) and called the right (respectively left) annihilator of $S$ in $R$. Instead of $r_{R}(\{a\})$ (respectively $l_{R}(\{a\})$, where $a$ is an element of $R$, we write simply $r_{R}(a)$ (respectively $l_{R}(a)$ ).

For background on the theory of radicals of rings we refer the reader to any standard text such as $[2,17]$. Recall that a radical $\mathscr{R}$ is called left (right) hereditary if $L<_{1} A$ $(H<r, A)$ and $A \in \mathscr{R}$ imply $L \in \mathscr{R}(H \in \mathscr{R})$. A radical which is left and right hereditary is called one-sided hereditary. A radical $\mathscr{R}$ is said to be left (right) strong if for every ring $A, \mathscr{R}(A)$ contains all left (right) $\mathscr{R}$-ideals of $A$. A radical which is left and right strong is called strong. The prime radical will be denoted by $\beta$.

\section{Properties of the singular ideal}

Let $M$ be a right module over a ring $R$. The singular submodule $Z_{R}(M)$ of $M$ is defined as $\left\{m \in M \mid \operatorname{ann}_{R}(m)\right.$ is an essential right ideal of $\left.R\right\}$.

The singular submodule of the right $R$-module $R_{R}$ is called the (right) singular ideal of the ring $R$ and denoted by $Z(R)$, that is, $Z(R)=\left\{x \in R \mid r_{R}(x) \cap H \neq 0\right.$ for every non-zero right ideal $H$ of $R$ \}. It is well known (see [7], p. 30) that $Z(R)$ is indeed an ideal of $R$.

Rings $R$ such that $Z(R)=R$ are called singular whereas those for which $Z(R)=0$ are called non-singular. We start with some examples of singular and non-singular rings.

PROPOSITION 2.1. Every commutative nil ring $R$ is singular:

PROOF. Take any $a \in R$ and $0 \neq b \in R$. If $n$ is the smallest natural number such that $a^{n} b R^{*}=0$, then $0 \neq a^{n-1} b R^{*} \subseteq r(a)$. However $a^{n-1} b R^{*}=b a^{n-1} R^{*} \subseteq b R^{*}$, so $b R^{*} \cap r(a) \neq 0$. Thus $Z(R)=R$. 
A ring $R$ is said to be (right) strongly prime [8] if every non-zero ideal $I$ of $R$ contains a finite subset $F$ such that $r_{R}(F)=0$. An ideal $P$ of a ring $A$ is called strongly prime if the ring $A / P$ is strongly prime.

\section{PROPOSITION 2.2. Every strongly prime ring $R$ is non-singular.}

PROOF. If $Z(R) \neq 0$, then since $Z(R) \triangleleft R$ and $R$ is right strongly prime, there exists a finite subset $F=\left\{f_{1}, \ldots, f_{n}\right\} \subseteq Z(R)$ such that $r\left(f_{1}\right) \cap \cdots \cap r\left(f_{n}\right)=r(F)=$ 0 . However $f_{i} \in Z(R)$, so $r\left(f_{i}\right)$ is an essential right ideal of $R$ for $i=1, \ldots, n$. Consequently $r(F)$ is an essential right ideal of $R$, a contradiction.

Let $R$ be a non-nilpotent finitely generated ring. For every natural number $n$, the ring $R^{n}$ is finitely generated as well. Hence applying Zorn's lemma one can find an ideal $M$ of $R$ maximal with respect to $R^{n} \nsubseteq M$ for $n=1,2, \ldots$ The ring $R / M$ is strongly prime. Indeed, if $I / M$ is a non-zero ideal of $R / M$, then the maximality of $M$ implies that there exists a natural number $n$, such that $R^{n} \subseteq I$. Let $F$ be a finite set generating the ring $R^{n}$. Then $r_{R / M}(F+M)=r_{R / M}\left(R^{n}+M / M\right)=J / M$ for an ideal $J$ of $R$. If $M \neq J$, then the maximality of $M$ implies that $R^{m} \subseteq J$ for a natural number $m$. However in this case $R^{n+m} \subseteq R^{n} J \subseteq M$, a contradiction.

Now from Proposition 2.2 it follows that the ring $R / M$ is non-singular. Taking in particular $R$ a finitely generated non-nilpotent nil ring (see [6]) one gets that $R / M$ is a non-singular nil ring. This shows that Proposition 2.1 does not hold for noncommutative nil rings.

Another class of non-singular rings is given by the following

PROPOSITION 2.3. Every reduced ring $R$ is non-singular.

PROOF. Take any $a \in R$. If $x \in r(a) \cap a R^{*}$, then for some $y \in R^{*}, x=a y$ and $a^{2} y=0$. This implies that $(a y R a)^{2}=0$. Hence, since $R$ is reduced, ay $R a=0$. Consequently $x R x=$ ay $R a y=0$. However the ring $R$ being reduced is semiprime, so $x=0$. Consequently $r(a) \cap a R^{*}=0$. This implies that $Z(R)=0$.

Note that for every family $\left\{R_{\alpha}\right\}$ of rings $Z\left(\Pi R_{\alpha}\right)=\Pi Z\left(R_{\alpha}\right)$ and $Z\left(\oplus R_{\alpha}\right)=$ $\oplus Z\left(R_{\alpha}\right)$. Hence the class of singular rings as well as the class of non-singular rings is closed under products and direct sums. However the classes are not closed under taking homomorphic images and ideals, as we will see in the following examples.

First note that the class of non-singular rings is not closed under taking homomorphic images. Indeed, the ring $x K[x]$ of polynomials with zero constant term over a field $K$ is a non-singular ring whereas its homomorphic image $x K[x] / x^{2} K[x]$ is a singular ring. 
EXAMPLE 2.4. The class of singular rings is not homomorphically closed.

Indeed, let $R$ be a commutative nil ring which is not nil of bounded index and let $P$ be the product of an infinite number of copies of $R$. By Proposition 2.1 and the foregoing remark, the ring $P$ is singular. Obviously $\beta(P) \neq P$. Since $P$ is commutative, the ring $P / \beta(P)$ is reduced. Hence the ring $P / \beta(P)$ is non-singular by Proposition 2.3.

Recall that a class $\mathscr{M}$ of rings is said to be hereditary if $I \triangleleft R, R \in \mathscr{M}$ imply $I \in \mathscr{M}$.

EXAMPLE 2.5. The class of singular rings is not hereditary.

Indeed, let $K$ be a field and $A$ a singular $K$-algebra such that $A^{2} \neq 0$. We claim that the ring $R=\left(\begin{array}{cc}A & A \\ 0 & A\end{array}\right)$ is singular. For, take any $a=\left(\begin{array}{l}x \\ 0 \\ 0\end{array}\right) \in R$. Clearly if $I=r_{A}(\{x, y, z\})$, then $\left(\begin{array}{ll}I & I \\ 0 & I\end{array}\right) \subseteq r_{R}(a)$. For every $0 \neq b=\left(\begin{array}{ll}b_{1} & b_{2} \\ 0 & b_{3}\end{array}\right) \in R, b R=\left(\begin{array}{ll}l_{1} & l_{2} \\ 0 & l_{3}\end{array}\right)$ for some right ideals $I_{1}, I_{2}, I_{3}$ of $A$. If some of $I_{i} \neq 0$ (or, equivalently, $b R \neq 0$ ), then since $Z(A)=A, I \cap I_{i} \neq 0$. Hence $0 \neq b R \cap\left(\begin{array}{l}I \\ 0\end{array}\right) \subseteq b R \cap r_{R}(a)$. Suppose that $b R=0$ and denote by $P$ the subring of $K$ generated by 1 . The right ideal of $R$ generated by $b$ is equal $P b$ and the right ideals of $A$ generated by $b_{i}$ are equal $P b_{i}$. Since $A=Z(A)$, there are non-zero elements $k_{1}, k_{2}, k_{3} \in P$ such that $k_{i} b_{i} \in I$, $i=1,2,3$. Putting $k=k_{1} k_{2} k_{3}$, we get that $0 \neq k b \in P b \cap\left(\begin{array}{l}l \\ 0\end{array}\right) \subseteq P b \cap r_{R}(a)$. Therefore for every $a \in R, r_{R}(a)$ is an essential right ideal of $R$. Thus $Z(R)=R$.

Now $J=\left(\begin{array}{cc}A & A \\ 0 & 0\end{array}\right) \triangleleft R$. Take $a \in A$ such that $H=r_{A}(a) \neq A$ (such an element exists because $A^{2} \neq 0$ ). Then there exists a non-zero $K$-subspace $V$ of $A$ with $H \cap V=0$. Note that $0 \neq\left(\begin{array}{cc}0 & V \\ 0 & 0\end{array}\right)<_{r} J$ and $\left(\begin{array}{cc}0 & V \\ 0 & 0\end{array}\right) \cap r_{J}\left(\begin{array}{cc}a & 0 \\ 0 & 0\end{array}\right)=\left(\begin{array}{cc}0 & V \\ 0 & 0\end{array}\right) \cap\left(\begin{array}{cc}H & H \\ 0 & 0\end{array}\right)=0$. Hence $a \notin Z(J)$.

From the above example it also follows that the class of singular rings is not closed under extensions. Indeed, the ring $J$ is not singular but both $I=\left(\begin{array}{cc}0 & A \\ 0 & 0\end{array}\right)$ and $J / I \simeq A$ are singular rings.

EXAMPLE 2.6. The class of non-singular rings is not hereditary.

Indeed, let $K$ be a field and $R=\left(\begin{array}{cc}K & 0 \\ K & 0\end{array}\right)$. Clearly $I=\left(\begin{array}{ll}0 & 0 \\ K & 0\end{array}\right) \triangleleft R$ and $Z(I)=I$. However $Z(R)=0$. In fact, for each $0 \neq x \in R, r_{R}(x)=I$ but $H=\left(\begin{array}{ll}K & 0 \\ 0 & 0\end{array}\right)<_{r} R$ and $I \cap H=0$. Thus $r_{R}(x)$ is not an essential right ideal of $R$.

The following proposition implies in particular that the class of semiprime nonsingular rings is hereditary.

PROPOSITION 2.7. If $I \triangleleft R$ and the ring $I$ is semiprime, then $Z(I)=I \cap Z(R)$. 
PROOF. Let $i \in Z(I)$ and $0 \neq H<_{r} R$. If $H I=0$, then $(I H)^{2}=0$. However $I$ is a semiprime ring and $I H<, I$, so $I H=0$. Consequently $H \subseteq r_{R}(I) \subseteq r_{R}(i)$. Thus in this case $H \cap r_{R}(i) \neq 0$. If $H I \neq 0$, then $0 \neq H I<_{r} I$. Hence, since $i \in Z(I), H I \cap r_{I}(i) \neq 0$. However $H I \subseteq H$ and $r_{I}(i) \subseteq r_{R}(i)$, so also in this case $H \cap r_{R}(i) \neq 0$. Therefore $i \in Z(R) \cap I$.

Now let $i \in I \cap Z(R)$ and $0 \neq H<_{r} I$. Since the ring $I$ is semiprime, $H I \neq 0$. Obviously $H I<_{r} R$. Hence $H I \cap r_{R}(i) \neq 0$. However $H I \subseteq H$, so $0 \neq H I \cap$ $r_{R}(i) \subseteq H \cap r_{R}(i)=H \cap r_{l}(i)$. This implies that $i \in Z(I)$.

\section{Behaviour of the singular ideal with respect to one-sided ideals}

Now we shall study the behaviour of $Z(\cdot)$ with respect to one-sided ideals. The main result of this section is the following

THEOREM 3.1. If $I$ is a one-sided ideal of a ring $R$ and $I^{*}$ is the two-sided ideal of $R$ generated by $I$, then $Z\left(I^{*} / \beta\left(I^{*}\right)\right) \neq 0$ if and only if $Z(I / \beta(I)) \neq 0$.

The proof of the theorem is split in several parts. We start with Lemma 3.2 which allows us to assume that $R$ is semiprime (then of course one has to prove that $Z\left(I^{*}\right) \neq 0$ if and only if $\left.Z(I / \beta(I)) \neq 0\right)$. In the semiprime case the result is the conjunction of Corollaries 3.7, 3.9 and Propositions 3.12, 3.13.

LEMMA 3.2. Let $K$ be a one-sided ideal of $A$ and let $K^{*}$ be the ideal of $A$ generated by $K$. Then $\bar{K}=(K+\beta(A)) / \beta(A)$ is a one-sided ideal of $A / \beta(A)$ and $\left(K^{*}+\right.$ $\beta(A)) / \beta(A)$ is the ideal of $A / \beta(A)$ generated by $\bar{K}$. Moreover $\left(K^{*}+\beta(A)\right) / \beta(A) \simeq$ $K^{*} / \beta\left(K^{*}\right)$ and $\bar{K} / \beta(\bar{K}) \simeq K / \beta(K)$.

PROOF. Straightforward.

Before passing to the semiprime case we shall prove the following

PROPOSITION 3.3. If $I<r$, then $Z(I) I \subseteq Z(R)$.

Proof. Take $z \in Z(I), i \in I$ and $0 \neq H<_{r}$. If $i H=0$, then $z i H=0$, and so in this case $H \subseteq r_{R}(z i)$. If $i H \neq 0$, then $0 \neq i H<_{r} I$. Hence, since $z \in Z(I)$, $i H \cap r_{l}(z) \neq 0$. Consequently in any case there is $0 \neq h \in H$ such that $z i h=0$. This implies that $H \cap r_{R}(z i) \neq 0$, so $z i \in Z(R)$.

LEMMA 3.4. If $R$ is a semiprime ring and $I<_{r} R\left(L<_{1} R\right)$, then $\beta(I) I=0$ $(L \beta(L)=0)$. 
Proof. Note that $\beta(I) I<_{r} R$ and $\beta(I) I \subseteq \beta(I)$. Hence $\beta(I) I$ is a right $\beta$-ideal of $R$. Since $R$ is semiprime, $\beta(I) I=0$.

\section{Proposition 3.3 and Lemma 3.4 give}

COROLLARY 3.5. If $R$ is a semiprime non-singular ring and $I<_{r} R$, then $Z(I)=$ $\beta(I)$.

Proof. By Proposition 3.3, $Z(I) I \subseteq Z(R)=0$. This obviously implies that $Z(I) \subseteq \beta(I)$. The converse inclusion is an immediate consequence of Lemma 3.4 and the definition of $Z(I)$.

PROPOSITION 3.6. If $R$ is a semiprime ring and $I<_{r} R$, then $(I \cap Z(R)+\beta(I)) / \beta(I)$ $\subseteq Z(I / \beta(I))$.

ProOF. Take $z \in I \cap Z(R)$ and $0 \neq H / \beta(I)<_{r} I / \beta(I)$. Clearly $0 \neq H I \subseteq H$ and $H I<_{r} R$. Hence $r_{R}(z) \cap H I \neq 0$. Since $R$ is semiprime and $H I \cap r_{R}(z)<_{r} R$, we have $H I \cap r_{I}(z)=H I \cap r_{R}(z) \nsubseteq \beta(I)$. Now $0 \neq\left(\left(H I \cap r_{I}(z)\right)+\beta(I)\right) / \beta(I) \subseteq$ $((H I+\beta(I)) / \beta(I)) \cap\left(\left(r_{I}(z)+\beta(I)\right) / \beta(I)\right) \subseteq(H I+\beta(I) / \beta(I)) \cap r_{I / \beta(I)}(z+\beta(I)) \subseteq$ $H / \beta(I) \cap r_{I / \beta(I)}(z+\beta(I))$. Hence $z+\beta(I) \in Z(I / \beta(I))$.

COROLlaRY 3.7. If $R$ is a semiprime ring, $I<_{r} R$ and $Z(I / \beta(I))=0$, then $Z\left(I^{*}\right)=0$, where $I^{*}=R^{*} I$.

PROOF. By Proposition 3.6, if $Z(I / \beta(I))=0$, then $I \cap Z(R) \subseteq \beta(I)$. Hence $I \cap Z(R)$ is a $\beta$-radical right ideal of $R$. However $R$ is a semiprime ring, so $I \cap Z(R)=$ 0 . Consequently $I Z(R)=0$. Thus by Proposition $2.7,\left(Z\left(I^{*}\right)\right)^{2} \subseteq R^{*} I Z\left(I^{*}\right) \subseteq$ $R^{*} I Z(R)=0$ and semiprimeness of $R$ implies that $Z\left(I^{*}\right)=0$.

PROPOSITION 3.8. If $R$ is a semiprime ring and $L<_{1} R$, then $L K \subseteq Z\left(L^{*}\right)$, where $L^{*}=L R^{*}$ and $K$ is the ideal of $L$ containing $\beta(L)$ such that $Z(L / \beta(L))=K / \beta(L)$.

PROOF. Let $0 \neq H<r L^{*}$. Suppose first that $H L \subseteq \beta(L)$. Then by Lemma 3.4, $L H L=0$ and hence $L H L^{*}=L H L R^{*}=0$. This gives $L H=0$, because $R$ is semiprime. Consequently $H \subseteq r_{L^{*}}(L) \subseteq r_{L^{*}}(L K)$. Suppose now that $H L \nsubseteq \beta(L)$. Then for every $k \in K,((H L+\beta(L)) / \beta(L)) \cap r_{L / \beta(L)}(k+\beta(L)) \neq 0$. Hence there is $t \in H L \backslash \beta(L)$ such that $k t \in \beta(L)$. By Lemma 3.4, Lkt $=0$, which gives $0 \neq t \in H L \cap r_{L^{*}}(L k) \subseteq H \cap r_{L^{*}}(L k)$.

The foregoing shows that for every $0 \neq H<, L^{*}$ and each $k \in K, H \cap r_{L^{\cdot}}(L k) \neq 0$. Hence $L K \subseteq Z\left(L^{*}\right)$. 
Proposition 3.8 gives

COROLlaRY 3.9. Under the assumptions of Proposition 3.8, if $Z(L / \beta(L)) \neq 0$, then $Z\left(L^{*}\right) \neq 0$.

It is much more complicated to get the symmetric versions of Corollaries 3.7 and 3.9. We begin with two auxiliary lemmas.

LEMMA 3.10. If $R$ is a semiprime ring and $0 \neq L<_{l} R$, then $L^{2} R^{*}$ is an essential ideal of $L R^{*}$.

PROOF. Suppose that $I \triangleleft L R^{*}$ and $I \cap L^{2} R^{*}=0$. Then $I L^{2} R^{*}=0$. However $I^{3} \subseteq I L R^{*} L R^{*}=I L^{2} R^{*}$. Hence $I=0$, because $R$ is semiprime.

LEMMA 3.11. (cf.[11], Lemma 2). For every element a of a ring $R$

(i) $I=r_{R}(a) a \triangleleft R a, J=a l_{R}(a) \triangleleft a R$;

(ii) $R a / I \simeq a R / J$;

(iii) $R a / \beta(R a) \simeq a R / \beta(a R)$.

PROOF. (i) Straightforward.

(ii) Note first that if for some $r_{1}, r_{2} \in R, r_{1} a=r_{2} a$, then $r_{1}-r_{2} \in l_{R}(a)$. Hence $a r_{1}+J=a r_{2}+J$. This implies that putting $f(r a)=a r+J$, we get a well defined map $f: R a \rightarrow a R / J$. Clearly $f\left(r_{1} a+r_{2} a\right)=f\left(r_{1} a\right)+f\left(r_{2} a\right)$. Now $f\left(r_{1} a r_{2} a\right)=a r_{1} a r_{2}+J=f\left(r_{1} a\right) f\left(r_{2} a\right)$. Hence $f$ is a ring homomorphism such that $f(R a)=a R / J$. It suffices to prove that $\operatorname{Ker} f=I$. The inclusion $I \subseteq \operatorname{Ker} f$ is clear. If $r a \in \operatorname{Ker} f$, then $a r \in J$. Hence there exists $r^{\prime} \in l_{R}(a)$ such that $a r=a r^{\prime}$. This implies that $r-r^{\prime} \in r_{R}(a)$ and $r a=\left(r-r^{\prime}\right) a \in I$. The proof is complete.

(iii) Note that $I^{2}=J^{2}=0$. Hence $I \subseteq \beta(R a), J \subseteq \beta(a R)$ and so $\beta(R a / I)=$ $\beta(R a) / I, \beta(a R / J)=\beta(a R) / J$. Consequently the isomorphism $R a / I \simeq a R / J$ induces an isomorphism of the rings $\beta(R a) / I$ and $\beta(a R) / J$ as well as an isomorphism of the rings $R a / \beta(R a)$ and $a R / \beta(a R)$. The result follows.

PROPOSITION 3.12. If $R$ is a semiprime ring, $L<_{1} R$ and $Z(L / \beta(L))=0$, then $Z\left(L^{*}\right)=0$, where $L^{*}=L R^{*}$.

Proof. Suppose that $Z\left(L^{*}\right) \neq 0$. By Lemma 3.4, $L^{2} R^{*}=\sum_{l \in L \backslash \beta(L)} L l R^{*}$. Obviously all $L I R^{*}$ are ideals of $L^{*}$. In a semiprime ring an ideal which intersects a sum of ideals must intersect one of them. Hence applying Lemma 3.10 and Proposition 2.7, we get that for some $l \in L \backslash \beta(L), Z\left(L l R^{*}\right) \neq 0$. Since $L l R^{*} \triangleleft R^{*} l R^{*}$, by Proposition 2.7, $Z\left(R^{*} l R^{*}\right) \neq 0$. Now $l R^{*}<, R$, so by Corollary $3.7, Z\left(l R^{*} / \beta\left(l R^{*}\right)\right) \neq 0$. Applying Lemma 3.11, we get that $l R^{*} / \beta\left(l R^{*}\right) \simeq R^{*} l / \beta\left(R^{*} l\right)$, so $Z\left(R^{*} l / \beta\left(R^{*} l\right)\right) \neq 0$. 
Now, since $R^{*} l<_{1} L$ and the ideal of $L$ generated by $R^{*} l$ is equal $R^{*} l+R^{*} l L$, Lemma 3.2 and Corollary 3.9 give $Z\left(\left(R^{*} l+R^{*} l L\right) / \beta\left(R^{*} l+R^{*} l L\right)\right) \neq 0$. However $R^{*} l+R^{*} l L \triangleleft L$, so by Proposition $2.7, Z(L / \beta(L)) \neq 0$. The result follows.

PROPOSITION 3.13. If $R$ is a semiprime ring, $I<_{r} R$ and $Z(I / \beta(I)) \neq 0$, then $Z\left(I^{*}\right) \neq 0$, where $I^{*}=R^{*} I$.

PROOF. Take $i \in I$ such that $0 \neq i+\beta(I) \in Z(I / \beta(I))$. Clearly $i R^{*}<_{r} I$, $J=i R^{*}+I i R^{*} \triangleleft I$ and $(J+\beta(I) / \beta(I)) \cap Z(I / \beta(I)) \neq 0$. Hence by Proposition $2.7, Z(J+\beta(I) / \beta(I)) \simeq Z(J / \beta(J)) \neq 0$. Since $J$ is the ideal of $I$ generated by $i R^{*}$, applying Lemma 3.2 and Corollary 3.7 , we get that $Z\left(i R^{*} / \beta\left(i R^{*}\right)\right) \neq 0$. Now by Lemma $3.11, i R^{*} / \beta\left(i R^{*}\right) \simeq R^{*} i / \beta\left(R^{*} i\right)$, so $Z\left(R^{*} i / \beta\left(R^{*} i\right)\right) \neq 0$. However $R^{*} i<I^{*}$, so by Corollary 3.9, $Z\left(R^{*} i+R^{*} i I^{*}\right) \neq 0$. Now Proposition 2.7 gives $Z\left(I^{*}\right) \neq 0$. The result follows.

\section{Radicals}

As we have mentioned in the introduction we would like to find a radical $\mathscr{R}$ such that for every ring $R, \mathscr{R}(R)$ is close to being a singular ring whereas $R / \mathscr{R}(R)$ is close to being a non-singular ring. Of course the best would be to have $\mathscr{R}(R)=Z(R)$. However $Z(\cdot)$ is not a radical. Namely there are rings $R$ for which $Z(R / Z(R)) \neq 0$.

EXAMPLE 4.1. Let $K$ be a field, $\gamma$ an ordinal number and $P_{\gamma}=K\left\{X_{\alpha} \mid \alpha<\gamma\right\} / I$, where $K\left\{X_{\alpha} \mid \alpha<\gamma\right\}$ is the $K$-algebra of polynomials with zero constant terms in non-commuting indeterminates $X_{\alpha}$ indexed by ordinal numbers $\alpha<\gamma$ and $I$ is the ideal of $K\left\{X_{\alpha} \mid \alpha<\gamma\right\}$ generated by the set $\left\{X_{\alpha_{1}} X_{\alpha_{2}} \mid \alpha_{1} \leq \alpha_{2}<\gamma\right\}$. Denote by $x_{\alpha}$ the image of $X_{\alpha}$ in $P_{\gamma}$. Observe that $x_{0} P_{\gamma}=0$ and $x_{0} P_{\gamma}^{*} \cap r_{P_{\gamma}}\left(x_{\alpha}\right)=0$ for all $\alpha>0$. These imply that $Z\left(P_{\gamma}\right)=\left(x_{0}\right)$, the ideal generated by $x_{0}$. Note that $P_{\gamma} /\left(x_{0}\right) \simeq P_{\gamma}$ when $\gamma$ is infinite and $P_{\gamma} /\left(x_{0}\right) \simeq P_{\gamma-1}$ when $\gamma$ is finite and greater than 1 . Hence $Z\left(P_{\gamma} / Z\left(P_{\gamma}\right)\right) \neq 0$ for $\gamma>1$.

Now we shall apply a construction similar to that of the lower Baer radical.

Given a ring $R$ we put $Z_{0}(R)=0$. For any ordinal $\alpha>0$ we define $Z_{\alpha}(R)$ by:

(i) If $\alpha=\delta+1$ is not a limit ordinal, then $Z_{\alpha}(R)$ is the ideal of $R$ such that $Z_{\alpha}(R) / Z_{\delta}(R)=Z\left(R / Z_{\delta}(R)\right)$;

(ii) If $\alpha$ is a limit ordinal, then $Z_{\alpha}(R)=\bigcup_{\delta<\alpha} Z_{\delta}(R)$.

There exists an ordinal $\rho$ such that $Z_{\rho}(R)=Z_{\gamma}(R)$ for each ordinal $\gamma>\rho$. We denote $Z_{\rho}(R)$ by $\mathscr{Z}(R)$ and call it the generalized singular ideal of $R$. 
For the ring $P_{\gamma}$ in Example 4.1, the chain $Z_{\alpha}\left(P_{\gamma}\right)$ stabilizes at $\gamma$ when $\gamma$ is a limit ordinal and at $\gamma-1$ otherwise. This in particular shows that the number of steps in which one gets the generalized singular ideal is not bounded.

As an immediate consequence of the definition of $\mathscr{Z}(R)$ we have that $\mathscr{Z}(R / \mathscr{Z}(R))$ $=0$. However $\mathscr{Z}(\cdot)$ still is not a radical. It follows from Example 2.4 which shows that the class $\{R \mid \mathscr{Z}(R)=R\}$ is not even homomorphically closed. The same example shows that the lower radical determined by the class of singular rings contains some domains, which are very far from being singular. Thus the lower radical determined by the class of singular rings is not of the type we are looking for.

There is another way for constructing in each ring $R$ an ideal $G(R)$ such that $R / G(R)$ is a non-singular ring ([16], Ch. 6, $\S 1$ and $\S 6)$. Consider $R / Z(R)$ as a right $R$-module and define $G(R)$ to be the right ideal of $R$ containing $Z(R)$ and such that $G(R) / Z(R)=Z_{R}(R / Z(R))$. We have the following (cf. [7], Ex. 20, p. 37 and [16], Proposition 6.2 in Ch. 6).

PROPOSITION 4.2. For every ring $R, G(R)$ is an ideal of $R$ such that $Z_{R}(R / G(R))$ $=0$ and $Z(R / G(R))=0$.

ProOF. If $a \in G(R)$, then $r_{R}(a+Z(R))=\{b \in R \mid a b \in Z(R)\}$ is an essential right ideal of $R$. Also, for any $r \in R, r_{R}(a+Z(R)) \subseteq r_{R}(r a+Z(R))$. Hence $r a \in G(R)$ and so $G(R)$ is an ideal of $R$.

Suppose that $a+G(R) \in Z_{R}(R / G(R))$ and $H$ is a non-zero right ideal of $R$. If $H \cap Z(R) \neq 0$, then since $a(H \cap Z(R)) \subseteq Z(R)$, there exists $0 \neq b \in H$ such that $a b \in Z(R)$. Suppose now that $H \cap Z(R)=0$ and take $0 \neq b \in H$ such that $a b \in G(R)$. Then $b \notin Z(R)$ and so there exists a non-zero right ideal $F$ of $R$ with $F \cap r_{R}(b)=0$. Also $r_{R}(a b+Z(R)) \cap F \neq 0$. Hence there exists $0 \neq c \in F$ such that $a b c \in Z(R)$. Since $b c \in H$ and $b c \neq 0$, we have that $r_{R}(a+Z(R)) \cap H \neq 0$ in any case. This shows that $a \in G(R)$ and consequently $Z_{R}(R / G(R))=0$.

Finally, if $a+G(R) \in Z(R / G(R))$, then $r_{R / G(R)}(a+G(R))$ is an essential right ideal of $R / G(R)$. Write $r_{R / G(R)}(a+G(R))=I / G(R)$. Then it is easy to see that $I$ is an essential right ideal of $R$. Since $r_{R}(a+G(R))=I$, it follows that $a+G(R) \in Z_{R}(R / G(R))=0$. Thus $Z(R / G(R))=0$ and the proof is complete.

The ideal $G(R)$ is reached in two steps, so it would be more convenient to use it instead of $\mathscr{Z}(R)$. However the following shows that $G(R)$ is larger than $\mathscr{Z}(R)$ and because of that not useful in constructing the desired radical.

PROPOSITION 4.3. For every ring $R, \mathscr{Z}(R) \subseteq G(R)$.

PROOF. Applying transfinite induction, it suffices to show that if $Z_{\gamma}(R) \subseteq G(R)$ and $\alpha=\gamma+1$, then $Z_{\alpha}(R) \subseteq G(R)$. 
Let $a \in Z_{\alpha}(R)$. Put $\bar{a}=a+G(R) \in R / G(R)$ and $r_{R}(\bar{a})=\{x \in R \mid a x \in G(R)\}$. Let $H$ be a non-zero right ideal of $R$. If $H \subseteq G(R)$, then since $G(R) \subseteq r_{R}(\bar{a})$, $r_{R}(\bar{a}) \cap H=H \neq 0$. If $H \nsubseteq G(R)$, then $H \nsubseteq Z_{\gamma}(R)$. In this case consider $\left(H+Z_{\gamma}(R)\right) / Z_{\gamma}(R)$ which is a non-zero right ideal of $R / Z_{\gamma}(R)$. Since $a+Z_{\gamma}(R) \in$ $Z\left(R / Z_{\gamma}(R)\right)$, there exists $b \in H+Z_{\gamma}(R)$ with $a b \in Z_{\gamma}(R)$ and $b \notin Z_{\gamma}(R)$. Thus we easily obtain $x \in H$ with $a x \in Z_{\gamma}(R) \subseteq G(R)$ and $x \neq 0$. This shows that $r_{R}(\bar{a}) \cap H \neq 0$ in any case and so $\bar{a} \in Z_{R}(R / G(R))$. Consequently $a \in G(R)$ and we are done.

It is easy to see that if $P_{\gamma}$ is the $K$-algebra of Example 4.1, then $\mathscr{Z}\left(P_{\gamma}^{*}\right)=P_{\gamma}$ and $G\left(P_{\gamma}^{*}\right)=P_{\gamma}^{*}$. This shows that in general $G(R)$ is strictly larger than $\mathscr{Z}(R)$.

Let $\mathscr{H}=\left\{R \mid\right.$ for every homomorphic image $R^{\prime}$ of $\left.R, \mathscr{Z}\left(R^{\prime}\right)=R^{\prime}\right\}$. It is not hard to see that $\mathscr{H}=\left\{R \mid\right.$ for every non-zero homomorphic image $R^{\prime}$ of $\left.R, Z\left(R^{\prime}\right) \neq 0\right\}$.

Obviously the class $\mathscr{H}$ is homomorphically closed. We also have

PROPOSITION 4.4. The class $\mathscr{H}$ is closed under extensions, that is, if $I \triangleleft R, I$ and $R / I$ are in $\mathscr{H}$, then $R$ is in $\mathscr{H}$.

PROOF. We shall prove first that for every non-zero ring $R$, if $I \triangleleft R, \mathscr{Z}(I)=I$ and $\mathscr{Z}(R / I)=R / I$, then $Z(R) \neq 0$. Suppose to the contrary that $Z(R)=0$. Then $I \neq 0$ and by Proposition 3.3, $Z(I) I=0$. Assume now that $I \subseteq J \triangleleft R, Z(I) J=0$ and $Z(R / J)=Z / J$. We claim that $Z(I) Z=0$. Indeed, let $z \in Z(I), a \in Z$ and $0 \neq H<{ }_{r} R$. Put $H^{\prime}=\{h \in H \mid a h \in J\}$. Obviously $H^{\prime}<_{r} R$. Moreover, since $a \in Z, H^{\prime} \neq 0$. Now, since $Z(I) J=0$, we have $z a H^{\prime}=0$. Hence $H^{\prime} \subseteq r_{R}(z a)$ which implies that $r_{R}(z a) \cap H \neq 0$. Consequently $z a \in Z(R)=0$, which proves the claim. By Zorn's Lemma there is an ideal $M$ such that $Z(I) M=0$ and $M$ is maximal for this. Let $Z / M=Z(R / M)$. By the foregoing $Z(I) Z=0$, so $M=R$. This obviously implies that $0 \neq Z(I) \subseteq Z(R)$, a contradiction.

Now to get the proposition it suffices to show that for every proper ideal $J$ of $R$, $Z(R / J) \neq 0$. Obviously $\mathscr{Z}(R /(I+J))=R /(I+J)$ and, since $(I+J) / J \simeq$ $I /(I \cap J), \mathscr{Z}((I+J) / J)=(I+J) / J$. Hence by the foregoing, $Z(R / J) \neq 0$.

The class $\mathscr{H}$ has already quite good properties (from the theory of radicals point of view) but still is not a radical class. Obviously nilpotent rings are in the class $\mathscr{H}$. Thus to show that the class is not radical it suffices to find a non-zero $\beta$-radical non-singular ring. The following shows that such rings exist.

EXAMPLE 4.5. Let $R$ be the ring of all infinite strictly upper triangular matrices which have only finitely many non-zero rows with entries in a field $K$. It is easy to check that $R \in \beta$. We shall show that $Z(R)=0$. Let $0 \neq a \in R$. If all entries of 
$a$ outside the $n$ 'th column are equal to zero, then $r_{R}(a)$ consists of all matrices in $R$ whose entries in the $n$-th row are equal to zero. The set $I$ of all matrices in $R$ whose entries outside the $n$-th row are equal to zero is a non-zero right ideal of $R$ such that $I \cap r_{R}(a)=0$. Thus $a \notin Z(R)$. Suppose now that $a$ is an arbitrary non-zero element in $R$ and the $(k, l)$-entry of $a$ is non-zero. Let $b$ be the matrix with the $(l, l+1)$ entry equal to 1 and all other entries equal to zero. Clearly $b \in R$ and $a b$ is a non-zero matrix in $R$ whose all entries except some in $l+1$-th column are equal to zero. Hence by the foregoing, $a b \notin Z(R)$ and so $a \notin Z(R)$. Consequently $Z(R)=0$.

Modifying slightly the definition of the class $\mathscr{H}$ we obtain a radical satisfying the earlier postulated conditions.

Let $\mathscr{S}=\{R \mid$ every non-zero homomorphic image of $R$ contains a non-zero ideal which is a singular ring $\}$. Applying Proposition 2.7 and the fact that for every ring $A, \beta(A) \neq 0$ if and only if $A$ contains a non-zero ideal $I$ with $I^{2}=0$ one obtains that $\mathscr{S}=\left\{R \mid\right.$ for every non-zero homomorphic image $R^{\prime}$ of $R, \beta\left(R^{\prime}\right) \neq 0$ or $\left.Z\left(R^{\prime}\right) \neq 0\right\}$. Equivalently $\mathscr{S}=\{R \mid R$ cannot be homomorphically mapped onto a non-zero semiprime non-singular ring $\}$. We shall call $\mathscr{S}$ the (right) singular radical.

From Proposition 2.7 it follows that the class of semiprime non-singular rings is weakly special, that is, is hereditary and such that if $I$ is in the class and $I$ is an essential ideal of $R$, then $R$ is in the class. Hence the general results concerning upper radicals give the following.

PROPOSITION 4.6. The singular radical is equal to the upper radical determined by the class of semiprime non-singular rings. It is a hereditary radical containing $\beta$.

Recall that the (right) strongly prime radical is defined $[8,10]$ as the upper radical determined by the class of (right) strongly prime rings. From Proposition 2.2 it follows that $\mathscr{S}$ is contained in the strongly prime radical. It is known that the (right) strongly prime radical is not one-sided hereditary [10] and it is right but not left strong [12]. We shall show that the singular radical is an $N$-radical, that is, contains $\beta$, is strong and one-sided hereditary.

\section{THEOREM 4.7. The singular radical is an $N$-radical.}

ProOF. Suppose that $0 \neq L<_{1} R$ and $L \in \mathscr{S}$. We shall show that $L^{*}=L R^{*} \in \mathscr{S}$. If $I$ is a semiprime ideal of $L^{*}$, then $I$ is an ideal of $R$. Hence $L^{*} / I=((L+I) / I) R^{*} / I$. This implies that if $I$ is a proper ideal of $L^{*}$, then $(L+I) / I \notin \beta$. Thus, since $L \in \mathscr{S}$, we have $Z(\bar{L} / \beta(\bar{L})) \neq 0$, where $\bar{L}=(L+I) / I$. Theorem 3.1 implies that $Z\left(L^{*} / I\right) \neq 0$. Consequently $L^{*} \in \mathscr{S}$, which proves that $\mathscr{S}$ is a left strong radical. Applying dual arguments and Theorem 3.1 one gets that $\mathscr{S}$ is a right strong radical.

We shall show now that if $L<_{1} R$ and $0 \neq R \in \mathscr{S}$, then $L \in \mathscr{S}$. Suppose that $I$ is a semiprime ideal of $L$ and let $M$ be an ideal of $R$ maximal with respect to $M \cap L \subseteq I$. 
Assume first that $M=0$. It is easy to see that $R$ is a semiprime ring and $L^{*}=L R^{*}$ is an essential ideal of $R$. Since $R \in \mathscr{S}, Z\left(L^{*}\right) \neq 0$ by Proposition 2.7. Now, $L I R \triangleleft R$ and $L I R L \subseteq I$. Hence $(L I R \cap L)^{2} \subseteq I$. However $I$ is a semiprime ideal of $L$, so $L I R \cap L \subseteq I$. This implies that $L I R=0$. Since the ring $R$ is semiprime, $L I=0$. This shows that $I=\beta(L)$. Hence by Theorem 3.1, $Z(L / I) \neq 0$.

If $M \neq 0$, then passing to the factor ring $R / M$ we reduce the situation to that considered above. This implies that $L \in \mathscr{S}$. Therefore $\mathscr{S}$ is a left hereditary radical. Applying dual arguments we get that $\mathscr{S}$ is right hereditary.

We do not know whether the radical $\mathscr{S}$ is special. Proposition 2.7 implies that the class $\mathscr{P}$ of prime non-singular rings is a special class. Thus the upper radical $\mathscr{S}^{\prime}$ determined by the class $\mathscr{P}$ is a special radical. We call it the special (right) singular radical. Clearly $\mathscr{S} \subseteq \mathscr{S}^{\prime}$. We raise the following

\section{QUESTION. Do the singular and special singular radicals coincide?}

Note that the above question is equivalent to the question of whether every semiprime non-singular ring can be homomorphically mapped onto a prime nonsingular ring.

One can check that $\mathscr{S}^{\prime}$ is an $N$-radical. The proof is very similar to that of Proposition 4.7 but one has to use in addition the following lemma.

LEMMA 4.8. If $R$ is a semiprime ring and $0 \neq L<_{l} R$ (respectively $0 \neq H<_{r} R$ ), then $L / \beta(L)$ (respectively $H / \beta(H)$ ) is a prime ring if and only if $L R^{*}$ (respectively $\left.R^{*} H\right)$ is a prime ring.

PRoOF. If $I_{1} / \beta(L)$ and $I_{2} / \beta(L)$ are non-zero ideals of $L / \beta(L)$, then $L I_{1} R$ and $L I_{2} R$ are non-zero ideals of $L R^{*}$ and $L I_{1} R L I_{2} R \subseteq L I_{1} I_{2} R$. Hence if $I_{1} I_{2} \subseteq \beta(L)$, then by Lemma 3.4, $L I_{1} R L I_{2} R=0$. This implies that if $L R^{*}$ is a prime ring, then $L / \beta(L)$ is a prime ring.

Conversely, suppose that $I_{1}$ and $I_{2}$ are non-zero ideals of $L R^{*}$ such that $I_{1} I_{2}=0$. Since $R$ is semiprime, $L R^{*} I_{i}^{2} L R^{*} \neq 0, i=1,2$. Now $L R^{*} I_{i}^{2} L R^{*}=L\left(R^{*} I_{i}\right) I_{i} L R^{*} \subseteq$ $L\left(L R^{*}\right) I_{i} L R^{*} \subseteq L I_{i} L R^{*}$, so $L I_{i} L \neq 0$ and by Lemma $3.4, I_{i} L \nsubseteq \beta(L), i=1,2$. However $I_{1} L I_{2} L \subseteq I_{1} I_{2}=0$. Hence $L / \beta(L)$ is not prime. The result follows.

In a number of papers (cf. [3] and the papers quoted therein) there were studied rings $R$ whose all prime homomorphic images are non-singular rings. Denote the class of all these rings by $\mathscr{T}$.

THEOREM 4.9. $\mathscr{T}$ is an $N$-radical. 
PROOF. Clearly the class $\mathscr{T}$ is homomorphically closed. Applying Proposition 2.7 one easily checks that every ring which is the union of a chain of $\mathscr{T}$-ideals is in $\mathscr{T}$. Suppose now that $I \triangleleft R$ and both $I$ and $R / I$ are in $\mathscr{T}$. Let $P$ be a prime ideal of $R$. If $I \subseteq P$, then $R / P$ is a homomorphic image of $R / I$. Hence $R / P$ is a non-singular ring. If $I \nsubseteq P$, then $\bar{I}=(I+P) / P$ is a non-zero ideal of the prime ring $\bar{R}=R / P$. By Proposition 2.7, $\bar{I} \cap Z(\bar{R})=Z(\bar{I})$. Since $\bar{I}$ is a prime homomorphic image of $I$ and $I$ is in $\mathscr{T}, Z(\bar{I})=0$. Consequently $Z(\bar{R})=0$ and it follows that $R$ is in $\mathscr{T}$. Therefore $\mathscr{T}$ is a radical.

Suppose that $L<_{1} R$ and $L$ is in $\mathscr{T}$. If $P$ is a prime ideal of $L^{*}=L R^{*}$, then $P$ is an ideal of $R$. Let $M$ be an ideal of $R$ maximal with respect to $M \cap L^{*}=P$. Then $M$ is a prime ideal of $R$ and $L^{*} / P \simeq \bar{L} \bar{R}^{*}$, where $\bar{L}=(L+M) / M$ and $\bar{R}=R / M$. By Lemma $4.8, \beta(\bar{L})$ is a prime ideal of $\bar{L}$. Hence $\bar{L} / \beta(\bar{L})$ is a non-singular ring. Applying Theorem 3.1 one gets that $L^{*} / P$ is a non-singular ring. This shows that $L^{*}$ is in $\mathscr{T}$. Consequently the radical $\mathscr{T}$ is left strong. Similarly, $\mathscr{T}$ is right strong.

Suppose now that $L<, R$ and $R$ is in $\mathscr{T}$. Let $P$ be a prime ideal of $L$ and $M$ be an ideal of $R$ maximal with respect to $M \cap L \subseteq P$. It is easy to see that $M$ is a prime ideal of $R$. Hence $R / M$ is a non-singular ring. Note that $\left(\left(L P R^{*}+M\right) \cap L\right)^{2} \subseteq P$. Since $P$ is a prime ideal of $L,\left(L P R^{*}+M\right) \cap L \subseteq P$. Consequently $L P R^{*} \subseteq M$ which easily implies that $(P+M) / M \subseteq \beta((L+M) / M)$. Now $(L+M) /(P+M)=$ $(L+P+M) /(P+M) \simeq L / L \cap(P+M)=L /(P+L \cap M)=L / P$. Thus $(P+M) / M=\beta((L+M) / M)$ and applying Proposition 2.7 and Theorem 3.1, we get that $(L+M) /(P+M)$ is a non-singular ring. Consequently $L / P$ is a non-singular ring. This shows that $L$ is in $\mathscr{T}$. Hence the class $\mathscr{T}$ is left hereditary. Similarly, $\mathscr{T}$ is right hereditary.

The proof is complete since clearly $\beta \subseteq \mathscr{T}$.

In [15] Snider proved that if $\mathscr{R}_{1}$ and $\mathscr{R}_{2}$ are hereditary radicals, then $\left(\mathscr{R}_{1}: \mathscr{R}_{2}\right)=$ $\left\{R \mid \mathscr{R}_{2}\left(R^{\prime}\right) \subseteq \mathscr{R}_{1}\left(R^{\prime}\right)\right.$ for every homomorphic image $R^{\prime}$ of $\left.R\right\}$ is the pseudocomplement of $\mathscr{R}_{2}$ relative to $\mathscr{R}_{1}$ in the lattice of all radicals, that is, $\left(\mathscr{R}_{1}: \mathscr{R}_{2}\right)$ is the largest among the radicals $\mathscr{R}$ satisfying $\mathscr{R} \cap \mathscr{R}_{2} \subseteq \mathscr{R}_{1}$.

Note that $\mathscr{T} \subseteq\left(\beta: \mathscr{S}^{\prime}\right)$. The radical $\left(\beta: \mathscr{S}^{\prime}\right)$ is an $N$-radical [13]. However it seems that the radicals $\mathscr{T}$ and $\left(\beta: \mathscr{S}^{\prime}\right)$ do not coincide.

\section{On rings satisfying the ascending chain condition on principal right annihilators}

It was proved in [9] that if $R$ is a ring satisfying the ascending chain condition on principal right annihilators, then $Z(R / \beta(R))=0$. This implies that for such rings $R$, $\mathscr{S}(R)=\beta(R)$. We shall show that the generalized singular ideal of rings of that type is contained in the prime radical. 
We start with the following

LEMMA 5.1. Let $\alpha$ be an ordinal number and $R$ a ring. If $a \in Z_{\alpha}(R)$ and $b \in$ $R \backslash Z_{\alpha}(R)$, then for some $x \in R^{*}, a x=0$ and $b x \neq 0$.

PROOF. If $\alpha=0$, then one can take $x=1$. Thus suppose $\alpha>1$ and, by transfinite induction, the results holds for smaller ordinals. If $\alpha=\gamma+1$ is not a limit ordinal, then there exists $t \in R$ such that at $\in Z_{\gamma}(R)$ and bt $\notin Z_{\gamma}(R)$. By the induction assumption there exists $x^{\prime} \in R^{*}$ such that $a t x^{\prime}=0$ and bt $x^{\prime} \neq 0$. Hence for $x=t x^{\prime}$ we have $a x=0$ and $b x \neq 0$. If $\alpha$ is a limit ordinal, then there exists $\gamma<\alpha$ such that $a \in Z_{\gamma}(R)$ and $b \in R \backslash Z_{\gamma}(R)$. Hence the result follows by the induction assumption.

PROPOSITION 5.2. If a ring $R$ satisfies the ascending chain condition on principal right annihilators, then $\mathscr{Z}(R) \subseteq \beta(R)$.

PROOF. Suppose that the result is not true and $\alpha$ is the smallest ordinal number for which $Z_{\alpha}(R) \nsubseteq \beta(R)$. Obviously $\alpha>0$ is not a limit ordinal. Suppose that $\alpha=\gamma+1$. Let $r(a)$ be a maximal ideal in the set $\left\{r(z) \mid z \in Z_{\alpha}(R) \backslash \beta(R)\right\}$. Since $a \notin \beta(R)$, there exists $x \in R$ such that axa $\notin \beta(R)$. Maximality of $r(a)$ implies that $r(a x a)=r(a)$. Since $a \notin \beta(R), a R \nsubseteq Z_{\gamma}(R)$. On the other hand $a x \in Z_{\alpha}(R)$ and $Z_{\alpha}(R) / Z_{\gamma}(R)=Z\left(R / Z_{\gamma}(R)\right)$, so there exists $y \in R$ such that $a y \notin Z_{\gamma}(R)$ and axay $\in Z_{\gamma}(R)$. Applying Lemma 5.1 we get that for some $t \in R^{*}$, ayt $\neq 0$ and axayt $=0$. This contradicts the equality $r(a x a)=r(a)$. The result follows.

Recall that an ideal $I$ of a ring $R$ is said to be right $T$-nilpotent if for every sequence $x_{1}, x_{2}, \ldots$ of elements of $I$ there exists an $n$ such that $x_{n} x_{n-1} \cdots x_{1}=0$.

PROPOSITION 5.3. If a ring $R$ satisfies the ascending chain condition on principal right annihilators, then $Z_{n}(R)$ is right $T$-nilpotent for every integer $n$.

PROOF. We proceed by induction on $n$. There is nothing to prove for $n=0$. Suppose that $n>0$ and $Z_{n-1}(R)$ is right $T$-nilpotent. Let $x_{1}, x_{2}, \ldots$ be a sequence of elements of $Z_{n}(R)$. If for every integer $l$ there exists $p$ such that $x_{l+p} \cdots x_{l} \in Z_{n-1}(R)$ we are done. Indeed, in this case there exist $m_{1}, m_{2}, \ldots$ with $x_{m_{1}} \cdots x_{1} \in Z_{n-1}(R)$, $x_{m_{1}+m_{2}} \cdots x_{m_{1}+1} \in Z_{n-1}(R), \ldots$. Hence there exist $t$ with $x_{m_{1}+\cdots+m_{i}} \cdots x_{1}=0$, since $Z_{n-1}(R)$ is $T$-nilpotent.

Thus assume that there exists an integer $l$ such that $x_{l+p} \cdots x_{l} \notin Z_{n-1}(R)$ for every $p>0$. Changing indices we can assume that $l=1$. Since $r\left(x_{1}\right) \subseteq r\left(x_{2} x_{1}\right) \subseteq \ldots$ is an ascending chain of principal right annihilators, there exists an $m$ with $r\left(x_{m} b\right)=r(b)$, $b=x_{m-1} \cdots x_{1}$. Now $x_{m} \in Z_{n}(R)$ and $b \notin Z_{n-1}(R)$, so there exists $y \in R^{*}$ with $x_{m}$ by $\in Z_{n-1}(R)$ and by $\notin Z_{n-1}(R)$. By Lemma 5.1 there exists $t \in R^{*}$ such that $x_{m} b y t=0$ and $b y t \neq 0$. Hence $r(b) \neq r\left(x_{m} b\right)$, a contradiction. 
The symmetric version of Proposition 1.5 in [5] shows that if a ring $R$ satisfies the ascending chain condition on right annihilators, then an ideal of $R$ is $T$-nilpotent if and only if it is nilpotent. Combining this result with Proposition 5.3 we obtain the following extension of Theorem 1.6 in [1].

COROLLARY 5.4. If $R$ is a ring satisfying the ascending chain condition on right annihilators, then $Z_{n}(R)$ is nilpotent for each integer $n$.

\section{References}

[1] A. W. Chatters and C. R. Hajarnavis, Rings with chain conditions (Pitman, Boston, 1980).

[2] N. J. Divinsky, Rings and radicals (Allen \& Unwin, 1965).

[3] M. Ferrero, 'Centred bimodules over prime rings: Closed submodules and applications to ring extensions', J. Algebra 172 (1995), 470-485.

[4] M. Ferrero and G. Törner, 'Rings with chain annihilator conditions and right distributive rings', Schriftenreihe des FB Mathematik, 190, Universität Duisburg, 1991.

[5] J. W. Fisher, 'On the nilpotency of nil subrings', Canad. J. Math. 23 (1970), 1211-1216.

[6] E. S. Golod, 'On nil algebras and finitely approximable groups', Izv. Akad. Nauk. SSSR Ser. Mat. 28 (1985), 273-276 (in Russian).

[7] K. R. Goodearl, Ring theory, nonsingular rings and modules (Marcel Dekker, New York, 1976).

[8] D. E. Handelman and J. Lawrence, 'Strongly prime rings', Trans. Amer. Math. Soc. 211 (1975), 209-233.

[9] B. Johns, 'Chain conditions and nil ideals', J. Algebra 73 (1981), 287-294.

[10] M. M. Parmenter, P. N. Stewart and R. Wiegandt, 'On the Groenewald-Heyman strongly prime radical', Quaestiones Math. 7 (1984), 225-240.

[11] E. R. Puczyłowski, 'Hereditariness of strong and stable radicals', Glasgow Math. J. 23 (1982), 85-90.

[12] — , 'On Sands' questions concerning strong and hereditary radicals', Glasgow Math. J. 28 (1986), 1-3.

[13] _ _ 'On the left hereditariness of pseudocomplement of hereditary radicals', Studia Sci. Math. Hungar. 27 (1992), 201-205.

[14] A. D. Sands, 'Radicals and Morita contexts', J. Algebra 24 (1973), 335-345.

[15] R. L. Snider, 'Lattices of radicals', Pacific. J. Math. 40 (1972), 207-220.

[16] W. Stenström, Rings of quotients (Springer, Berlin, 1975).

[17] R. Wiegandt, Radical and semisimple classes of rings, Queen's Papers in Pure and Appl. Math. No. 37 (Kingston, Ontario, 1974).

Instituto de Matemática

Universidade Federal do Rio Grande do Sul

91509-900 Porto Alegre

Brazil

email: ferrero@mat.ufrgs.br
Institute of Mathematics

University of Warsaw

02-097 Warsaw, Banacha 2

Poland

email: edmundp@mimuw.edu.pl 